Bull. Korean Math. Soc. 52 (2015), No. 2, pp. 531-540

http://dx.doi.org/10.4134/BKMS.2015.52.2.531

\title{
AN INDEPENDENT RESULT FOR ATTACHED PRIMES OF CERTAIN TOR-MODULES
}

\author{
Pham HuU Khanh
}

\begin{abstract}
Let $(R, \mathfrak{m})$ be a Noetherian local ring, $I$ an ideal of $R$, and $A$ an Artinian $R$-module. Let $k \geq 0$ be an integer and $r=\operatorname{Width}_{>k}(I, A)$ the supremum of length of $A$-cosequence in dimension $>k$ in $I$ defined by Nhan-Hoang [8]. It is shown that for all $t \leq r$ the sets

$$
\begin{gathered}
\left(\bigcup_{i=0}^{t} \operatorname{Att}_{R}\left(\operatorname{Tor}_{i}^{R}\left(R / I^{n}, A\right)\right)\right)_{\geq k} \text { and } \\
\left(\bigcup_{i=0}^{t} \operatorname{Att}_{R}\left(\operatorname{Tor}_{i}^{R}\left(R /\left(a_{1}^{n_{1}}, \ldots, a_{l}^{n_{l}}\right), A\right)\right)\right)_{\geq k}
\end{gathered}
$$

are independent of the choice of $n, n_{1}, \ldots, n_{l}$ for any system of generators $\left(a_{1}, \ldots, a_{l}\right)$ of $I$.
\end{abstract}

\section{Introduction}

Throughout this paper, let $(R, \mathfrak{m})$ be a Noetherian local ring, $I$ an ideal of $R$. Let $M$ be a finitely generated $R$-module, and $A$ an Atinian $R$-module. For a subset $T$ of $\operatorname{Spec}(R)$ and an integer $i \geq 0$, denote by $(T)_{\geq i}$ the set of all prime ideals $\mathfrak{p} \in T$ such that $\operatorname{dim} R / \mathfrak{p} \geq i$.

In 1976, L. J. Ratliff [10] asked whether the set of associated prime ideals $\operatorname{Ass}_{R}\left(R / I^{n}\right)$ is stable for large $n$. An affirmative answer was given by Ratliff [10] for the case $R$ is an domain. M. Brodmann [1] answered fully Ratliff's question not only for ring $R$, but also for module $M$. He proved that the sets $\operatorname{Ass}_{R}\left(M / I^{n} M\right)$ and $\operatorname{Ass}_{R}\left(I^{n} M / I^{n+1} M\right)$ are stable for large $n$. As dual results, R. Y. Sharp [12] proved that the sets of attached prime ideals $\operatorname{Att}_{R}\left(0:_{A} I^{n}\right)$ and $\operatorname{Att}_{R}\left(\left(0:{ }_{A} I^{n+1}\right) /\left(0:_{A} I^{n}\right)\right)$ are stable for large $n$. Starting from the observation that $M / I^{n} M \cong \operatorname{Tor}_{0}^{R}\left(R / I^{n}, M\right)$ and $\left(0:_{A} I^{n}\right) \cong \operatorname{Ext}_{R}^{0}\left(R / I^{n}, A\right)$, L. Melkersson and P. Schenlzel [6] developed the above results of Brodmann and Sharp.

Received February 21, 2014; Revised May 15, 2014.

2010 Mathematics Subject Classification. 13D45, 13E05.

Key words and phrases. asymptotic stability, attached prime, Tor-module, $A$-cosequence in dimension $>k$, width in dimension $>k$.

The author is supported by the Vietnam National Foundation for Science and Technology Development (Nafosted). 
They proved that the sets $\operatorname{Ass}_{R}\left(\operatorname{Tor}_{i}^{R}\left(R / I^{n}, M\right)\right)$ and $\operatorname{Att}_{R}\left(\operatorname{Ext}_{R}^{i}\left(R / I^{n}, A\right)\right)$ do not depend on $n$ for large $n$. In that paper, Melkersson and Schenlzel asked whether the sequence $\operatorname{Att}_{R}\left(\operatorname{Tor}_{i}^{R}\left(R / I^{n}, A\right)\right)$ becomes constant for large $n$.

However, Katzman [4, Corollary 1.3] constructed an example of local ring $(R, \mathfrak{m})$ of dimension 5 and two elements $x, y \in \mathfrak{m} \operatorname{such}$ that $\operatorname{Ass}_{R}\left(H_{(x, y) R}^{2}(R)\right)$ is an infinite set. Therefore, the set $\bigcup_{n \in \mathbb{N}} \operatorname{Ass}_{R}\left(\operatorname{Ext}_{R}^{2}\left(R / I^{n}, R\right)\right)$ is infinite, where $I=(x, y) R$. Hence, $\bigcup_{n \in \mathbb{N}} \operatorname{Att}_{R}\left(\operatorname{Tor}_{2}^{R}\left(R / I^{n}, E\right)\right)$ is infinite, where $E$ in the injective envelope of $R / \mathfrak{m}$ which is an Artinian $R$-module. It implies that the set $\operatorname{Att}_{R}\left(\operatorname{Tor}_{2}^{R}\left(R / I^{n}, E\right)\right)$ is not stable for large $n$. Therefore, ones need to find the conditions for the set $\bigcup_{n \in \mathbb{N}} \operatorname{Att}_{R}\left(\operatorname{Tor}_{i}^{R}\left(R / I^{n}, A\right)\right)$ to be finite.

Following Nhan-Hoang [8], a sequence $\left(x_{1}, \ldots, x_{r}\right)$ of elements in $\mathfrak{m}$ is called an $A$-cosequence in dimension $>k$ if $x_{i} \notin \mathfrak{p}$ for all $\mathfrak{p} \in\left(\operatorname{Att}_{R}\left(0:_{A}\left(x_{1}, \ldots\right.\right.\right.$, $\left.\left.\left.x_{i-1}\right) R\right)\right)_{>k}$ for every $i=1, \ldots, r$. Then in [7], Nhan-Dung proved that the set

$$
\left(\bigcup_{n \in \mathbb{N}} \operatorname{Att}_{R}\left(\operatorname{Tor}_{t}^{R}\left(R / I^{n}, A\right)\right)\right)_{\geq k}
$$

is finite for all $t \leq r$, where $r$ is the maximal length of an $A$-cosequence in dimension $>k$ in $I$. Moreover, they also proved that

$$
\left(\bigcup_{n_{1}, \ldots, n_{l} \in \mathbb{N}} \operatorname{Att}_{R}\left(\operatorname{Tor}_{t}^{R}\left(R /\left(a_{1}^{n_{1}}, \ldots, a_{l}^{n_{l}}\right), A\right)\right)\right)_{\geq k}
$$

is finite for all $t \leq r$, for any system of generators $\left(a_{1}, \ldots, a_{l}\right)$ of $I$.

The main result of this paper is the following theorem.

Theorem 1.1. Let $k \geq 0$ be an integer and $r=\operatorname{Width}_{>k}(I, A)$. Then for all $t \leq r$ we have

$$
\begin{aligned}
\left(\bigcup_{i=0}^{t} \operatorname{Att}_{R}\left(\operatorname{Tor}_{i}^{R}(R / I, A)\right)\right)_{\geq k} & =\left(\bigcup_{i=0}^{t} \operatorname{Att}_{R}\left(\operatorname{Tor}_{i}^{R}\left(R / I^{n}, A\right)\right)\right)_{\geq k} \\
& =\left(\bigcup_{i=0}^{t} \operatorname{Att}_{R}\left(\operatorname{Tor}_{i}^{R}\left(R /\left(a_{1}^{n_{1}}, \ldots, a_{l}^{n_{l}}\right), A\right)\right)\right)_{\geq k}
\end{aligned}
$$

for all $n, n_{1}, \ldots, n_{l} \in \mathbb{N}$ and for any system of generators $\left(a_{1}, \ldots, a_{l}\right)$ of $I$.

It should be mentioned that Theorem 1.1 shows that for all $t \leq r$ the sets

$$
\left(\bigcup_{i=0}^{t} \operatorname{Att}_{R}\left(\operatorname{Tor}_{i}^{R}\left(R / I^{n}, A\right)\right)\right)_{\geq k} \text { and }\left(\bigcup_{i=0}^{t} \operatorname{Att}_{R}\left(\operatorname{Tor}_{i}^{R}\left(R /\left(a_{1}^{n_{1}}, \ldots, a_{l}^{n_{l}}\right), A\right)\right)\right)_{\geq k}
$$

are independent of the choice of $n, n_{1}, \ldots, n_{l}$ for any system of generators $\left(a_{1}, \ldots, a_{l}\right)$ of $I$. As a consequence, we get again the conclusion of Nhan-Dung [7, Corollary 3.6], which says that if $k=-1$, then the sets

$$
\operatorname{Att}_{R}\left(\operatorname{Tor}_{r}^{R}\left(R / I^{n}, A\right)\right) \text { and } \operatorname{Att}_{R}\left(\operatorname{Tor}_{r}^{R}\left(R /\left(a_{1}^{n_{1}}, \ldots, a_{l}^{n_{l}}\right), A\right)\right)
$$


are independent of the choice of $n, n_{1}, \ldots, n_{l}$ for any system of generators $\left(a_{1}, \ldots, a_{l}\right)$ of $I$, where $r=\operatorname{Width}(I, A)$ is the maximal length of a $A$-cosequence in $I$ (see Proposition 3.8).

This paper is divided into three sections. In the next section, we show some auxiliary lemmas. The last section devotes to prove the main result of this paper, Theorem 1.1 (see Theorem 3.4).

\section{Preliminaries}

The theory of secondary representation of a module was introduced by I. G. Macdonald [5], which is in some sense dual to the theory of primary decomposition. Following [5], any Artinian $R$-module $A$ has a minimal secondary representation $A=A_{1}+\cdots+A_{r}$, where $A_{i}$ is $\mathfrak{p}_{i}$-secondary. The set $\left\{\mathfrak{p}_{1}, \ldots, \mathfrak{p}_{r}\right\}$ is independent of the choice of a minimal secondary representation of $A$, and it is denoted by $\operatorname{Att}_{R}(A)$.

Next, we recall the definition of $A$-cosequence in dimension $>k$ which was first given by Nhan and Hoang in [8, Definition 2.4].

Definition 2.1. Let $k \geq-1$ be an integer. A sequence $\left(x_{1}, \ldots, x_{r}\right)$ of elements in $\mathfrak{m}$ is called an $A$-cosequence in dimension $>k$ if $x_{i} \notin \mathfrak{p}$ for all $\mathfrak{p} \in\left(\operatorname{Att}_{R}\left(0:_{A}\right.\right.$ $\left.\left.\left(x_{1}, \ldots, x_{i-1}\right) R\right)\right)_{>k}$ for every $i=1, \ldots, r$.

By Nhan and Dung [7, Theorem 2.8], if $\operatorname{dim}_{R}\left(0::_{A} I\right)>k$, then any $A$ cosequence in dimension $>k$ in $I$ can be extended to a maximal one, and all maximal $A$-cosequences in dimension $>k$ in $I$ has the same length. This common length is called the width in dimension $>k$ in $I$ with respect to $A$ and denoted by $\operatorname{Width}_{>k}(I, A)$. If $\operatorname{dim}_{R}\left(0:_{A} I\right) \leq k$, then for every positive integer $r$, we can choose an $A$-cosequence in dimension $>k$ in $I$ of length $r$, in this case we set $\operatorname{Width}_{>k}(I, A)=+\infty$. Note that the width in dimension $>-1$ in $I$ with respect to $A$ exactly the width of $A$ in $I$ defined by A. Ooishi $[9]$ and it is denoted by $\operatorname{Width}(I, A)$.

For an ideal $I$ of $R$, denote by $\operatorname{Var}(I)$ the set of all prime ideals $\mathfrak{p}$ of $R$ containing $I$.

Lemma $2.2([5])$. The set of all minimal elements of $\operatorname{Att}_{R}(A)$ is exactly the set of all minimal elements of $\operatorname{Var}\left(\operatorname{Ann}_{R}(A)\right)$. Moreover,

$$
\operatorname{dim}_{R}(A)=\max \left\{\operatorname{dim}(R / \mathfrak{p}) \mid \mathfrak{p} \in \operatorname{Att}_{R}(A)\right\} .
$$

Note that $A$ has a natural structure as an $\widehat{R}$-module. With this structure, a subset of $A$ is an $R$-submodule if and only if it is an $\widehat{R}$-submodule. Therefore $A$ is an Artinian $\widehat{R}$-module.

The following results are well-known and will be used in the sequel.

Lemma 2.3 ([2, 8.24 and 8.25]). $\operatorname{Att}_{R}(A)=\left\{\widehat{\mathfrak{p}} \cap R \mid \widehat{\mathfrak{p}} \in \operatorname{Att}_{\widehat{R}}(A)\right\}$. 
Lemma 2.4. Let $f: R \rightarrow R^{\prime}$ be a ring homomorphism and $L$ an $R^{\prime}$-module. Consider $L$ as an $R$-module induced by $f$. Then we have

$$
\operatorname{Ass}_{R}(L)=\left\{f^{-1}(\mathfrak{P}) \mid \mathfrak{P} \in \operatorname{Ass}_{R^{\prime}}(L)\right\} .
$$

Denote by $E=E(R / \mathfrak{m})$ the injective envelope of $R / \mathfrak{m}$. The Matlis dual $D(A)$ of $A$ is defined by $D(A)=\operatorname{Hom}_{R}(A, E)$. Note that $D(A)=\operatorname{Hom}_{R}(A, E)$ $=\operatorname{Hom}_{\widehat{R}}(A, E)$. Moreover, $D(A)$ is a finitely generated $\widehat{R}$-module and by Sharp [11, Theorem 2.3] we have

$$
\operatorname{Ass}_{\widehat{R}}(D(A))=\operatorname{Att}_{\widehat{R}}(A) .
$$

Now we need to introduce the notion of poor filter sequence. An element $x \in R$ is called an $M$-poor filter regular if $x \notin \mathfrak{p}$ for all $\mathfrak{p} \in \operatorname{Ass}_{R}(M) \backslash\{\mathfrak{m}\}$. A sequence $x_{1}, \ldots, x_{r}$ of elements in $R$ is called an $M$-poor filter sequence if $x_{i}$ is $\left(M /\left(x_{1}, \ldots, x_{i-1}\right) M\right)$-poor filter regular for all $i=1, \ldots, r$. It is clear that a sequence $x_{1}, \ldots, x_{r}$ of elements in $R$ is an $M$-filter sequence in sense of Cuong-Schenzel-Trung [3] if and only if it is an $M$-poor filter sequence and $x_{1}, \ldots, x_{r} \in \mathfrak{m}$.

Lemma 2.5. Let $k \geq 0$ be an integer. Assume that $\left(x_{1}, \ldots, x_{r}\right)$ is an $A$ cosequence in dimension $>k$. Then $\left(\bar{x}_{1}, \ldots, \bar{x}_{r}\right)$ is a $D(A)_{\widehat{p}}$-poor filter sequence for all $\widehat{\mathfrak{p}} \in \operatorname{Var}\left(\operatorname{Ann}_{\widehat{R}} A\right)$ satisfying $\operatorname{dim}(R /(\widehat{\mathfrak{p}} \cap R)) \geq k$. Here $\bar{x}_{i}$ is the image of $x_{i}$ in $\widehat{R}_{\widehat{p}}$ for $i=1, \ldots, r$.

Proof. Let $\left(x_{1}, \ldots, x_{r}\right)$ be an $A$-cosequence in dimension $>k$. Assume that there exists $\widehat{\mathfrak{p}} \in \operatorname{Var}\left(\operatorname{Ann}_{\widehat{R}} A\right)$ satisfying $\operatorname{dim}(R /(\widehat{\mathfrak{p}} \cap R)) \geq k$ such that $\left(\bar{x}_{1}, \ldots\right.$, $\left.\bar{x}_{r}\right)$ is not an $D(A)_{\widehat{\mathfrak{p}}}$-poor filter sequence. Then $\bar{x}_{i} \in \widehat{\mathfrak{q}} \widehat{R}_{\widehat{\mathfrak{p}}}$ for some $i=1, \ldots, r$ and some $\widehat{\mathfrak{q}} \widehat{R}_{\widehat{\mathfrak{p}}} \in \operatorname{Ass}_{\widehat{R}}\left(D(A)_{\widehat{\mathfrak{p}}} /\left(x_{1}, \ldots, x_{i-1}\right) D(A)_{\widehat{\mathfrak{p}}}\right), \widehat{\mathfrak{q}} \widehat{R}_{\widehat{\mathfrak{p}}} \mp \widehat{\mathfrak{p}} \widehat{R}_{\widehat{\mathfrak{p}}}$.

Since $\widehat{\mathfrak{q}} \in \operatorname{Ass}_{\widehat{R}}\left(D(A) /\left(x_{1}, \ldots, x_{i-1}\right) D(A)\right)$, we have $\widehat{\mathfrak{q}} \in \operatorname{Att}_{\widehat{R}}\left(0:_{A}\left(x_{1}, \ldots\right.\right.$, $\left.\left.x_{i-1}\right) \widehat{R}\right)$. It follows by Lemma 2.3 that $\widehat{\mathfrak{q}} \cap R \in \operatorname{Att}_{R}\left(0:_{A}\left(x_{1}, \ldots, x_{i-1}\right) R\right)$. As $\widehat{\mathfrak{q}} \varsubsetneqq \widehat{\mathfrak{p}}$ and $\bar{x}_{i} \in \widehat{\mathfrak{q}} \widehat{R}_{\widehat{\mathfrak{p}}}$ we have $x_{i} \in \widehat{\mathfrak{q}} \cap R$ and $\operatorname{dim}(R / \widehat{\mathfrak{q}} \cap R)>\operatorname{dim}(R / \widehat{\mathfrak{p}} \cap R) \geq k$. This gives a contradiction.

\section{Main results}

First of all we recall some auxiliary lemmas. The following result is wellknown.

Lemma 3.1. Let $r=\operatorname{depth}(I, M)$ and $x_{1}, \ldots, x_{r}$ be an $M$-regular sequence. Then

$$
\begin{aligned}
\operatorname{Ass}_{R}\left(\operatorname{Ext}_{R}^{r}(R / I, M)\right) & =\operatorname{Ass}_{R}\left(H_{I}^{r}(M)\right) \\
& =\operatorname{Ass}_{R}\left(M /\left(x_{1}, \ldots, x_{r}\right) M\right) \cap \operatorname{Var}(I) .
\end{aligned}
$$


Remark 3.2. Assume that $r=\operatorname{depth}(I, M)$. Since $\operatorname{rad}(I)=\operatorname{rad}\left(I^{n}\right)=\operatorname{rad}\left(a_{1}^{n_{1}}\right.$, $\left.\ldots, a_{l}^{n_{l}}\right)$, where $\left(a_{1}, \ldots, a_{l}\right)$ is a system of generators of $I$, we have

$$
\begin{aligned}
\operatorname{Ass}_{R}\left(\operatorname{Ext}_{R}^{r}\left(R / I^{n}, M\right)\right) & =\operatorname{Ass}_{R}\left(H_{I^{n}}^{r}(M)\right) \\
& =\operatorname{Ass}_{R}\left(H_{I}^{r}(M)\right)=\operatorname{Ass}_{R}\left(\operatorname{Ext}_{R}^{r}(R / I, M)\right) . \\
\operatorname{Ass}_{R}\left(\operatorname{Ext}_{R}^{r}\left(R /\left(a_{1}^{n_{1}}, \ldots, a_{l}^{n_{l}}\right)^{n}, M\right)\right) & =\operatorname{Ass}_{R}\left(H_{I}^{r}(M) .\right.
\end{aligned}
$$

Moreover, by the proof of [7, Main Theorem], we have the following lemma.

Lemma 3.3. Let $k \geq 0$ be an integer and $r=\operatorname{Width}_{>k}(I, A)$. Then, for all $t \leq r$, we have $\mathbb{N}$.

(i) $\left(\bigcup_{i=0}^{t} \operatorname{Att}_{R}\left(\operatorname{Tor}_{i}^{R}\left(R / I^{n}, A\right)\right)\right)_{\geq k} \subseteq\left(\bigcup_{i=0}^{t} \operatorname{Att}_{R}\left(\operatorname{Tor}_{i}^{R}(R / I, A)\right)\right)_{\geq k}$ for all $n \in$

(ii) $\left(\bigcup_{i=0}^{t} \operatorname{Att}_{R}\left(\operatorname{Tor}_{i}^{R}\left(R /\left(a_{1}^{n_{1}}, \ldots, a_{l}^{n_{l}}\right), A\right)\right)\right)_{\geq k} \subseteq\left(\bigcup_{i=0}^{t} \operatorname{Att}_{R}\left(\operatorname{Tor}_{i}^{R}(R / I, A)\right)\right)_{\geq k}$ for any system of generators $\left(a_{1}, \ldots, a_{l}\right)$ of $I$ and for all $n_{1}, \ldots, n_{l} \in \mathbb{N}$.

Now we present the main result of this paper.

Theorem 3.4. Let $k \geq 0$ be an integer and $r=\operatorname{Width}_{>k}(I, A)$. Then for all $t \leq r$ we have

$$
\begin{aligned}
\left(\bigcup_{i=0}^{t} \operatorname{Att}_{R}\left(\operatorname{Tor}_{i}^{R}(R / I, A)\right)\right)_{\geq k} & =\left(\bigcup_{i=0}^{t} \operatorname{Att}_{R}\left(\operatorname{Tor}_{i}^{R}\left(R / I^{n}, A\right)\right)\right)_{\geq k} \\
& =\left(\bigcup_{i=0}^{t} \operatorname{Att}_{R}\left(\operatorname{Tor}_{i}^{R}\left(R /\left(a_{1}^{n_{1}}, \ldots, a_{l}^{n_{l}}\right), A\right)\right)\right)_{\geq k}
\end{aligned}
$$

for all $n, n_{1}, \ldots, n_{l} \in \mathbb{N}$ and for any system of generators $\left(a_{1}, \ldots, a_{l}\right)$ of $I$.

Proof. We first claim that

$\left(\bigcup_{i=0}^{t} \operatorname{Att}_{R}\left(\operatorname{Tor}_{i}^{R}(R / I, A)\right)\right)_{\geq k}=\left(\bigcup_{i=0}^{t} \operatorname{Ass}_{R}\left(D(A) /\left(x_{1}, \ldots, x_{i}\right) D(A)\right)\right)_{\geq k} \cap \operatorname{Var}(I)$

for all $t \leq r$, where $\left(x_{1}, \ldots, x_{r}\right)$ is an $A$-cosequence in dimension $>k$ in $I$.

Let $\mathfrak{p} \in\left(\bigcup_{i=0}^{t} \operatorname{Att}_{R}\left(\operatorname{Tor}_{i}^{R}(R / I, A)\right)\right)_{\geq k}$. We take an integer $i_{0} \leq t$ such that

$$
\mathfrak{p} \in \operatorname{Att}_{R}\left(\operatorname{Tor}_{i_{0}}^{R}(R / I, A)\right) \text { and } \mathfrak{p} \notin \operatorname{Att}_{R}\left(\operatorname{Tor}_{i}^{R}(R / I, A)\right)
$$

for all $i<i_{0}$.

Since $\mathfrak{p} \in \operatorname{Att}_{R}\left(\operatorname{Tor}_{i_{0}}^{R}(R / I, A)\right)$, by Lemma 2.3 there exists

$$
\widehat{\mathfrak{p}} \in \operatorname{Att}_{\widehat{R}}\left(\operatorname{Tor}_{i_{0}}^{R}(R / I, A)\right)
$$

such that $\widehat{\mathfrak{p}} \cap R=\mathfrak{p}$. Hence $\operatorname{Ann}_{\widehat{R}}(A) \subseteq \widehat{\mathfrak{p}}$ and $I \widehat{R} \subseteq \widehat{\mathfrak{p}}$. Since $\operatorname{dim} R /(\widehat{\mathfrak{p}} \cap R)=$ $\operatorname{dim} R / \mathfrak{p} \geq k$, we get by Lemma 2.5 that $\left(\bar{x}_{1}, \ldots, \bar{x}_{i_{0}}\right)$ is a $D(A)_{\hat{\mathfrak{p}}}$-poor filter 
sequence. Since $x_{1}, \ldots, x_{i_{0}} \in I$ and $I \widehat{R} \subseteq \widehat{\mathfrak{p}}$, it follows that $\left(\bar{x}_{1}, \ldots, \bar{x}_{i_{0}}\right)$ is a $D(A)_{\widehat{\mathfrak{p}}}$-filter sequence.

If $\mathfrak{p} \notin \operatorname{Ass}_{R}\left(D(A) /\left(x_{1}, \ldots, x_{i}\right) D(A)\right)$ for all $i<i_{0}$, then

$$
\widehat{\mathfrak{p}} \widehat{R}_{\widehat{\mathfrak{p}}} \notin \operatorname{Ass}_{\widehat{R_{\mathfrak{p}}}}\left(D(A)_{\widehat{\mathfrak{p}}} /\left(\bar{x}_{1}, \ldots, \bar{x}_{i}\right) D(A)_{\widehat{\mathfrak{p}}}\right)
$$

for all $i<i_{0}$. It implies that $\left(\bar{x}_{1}, \ldots, \bar{x}_{i_{0}}\right)$ is a $D(A)_{\widehat{p}^{-r e g u l a r ~ s e q u e n c e ~ a n d ~ w e ~}}$ have $\operatorname{depth}\left(I \widehat{R}_{\widehat{\mathfrak{p}}}, D(A)_{\widehat{\mathfrak{p}}}\right) \geq i_{0}$.

On the other hand, since $\widehat{\mathfrak{p}} \in \operatorname{Att}_{\widehat{R}}\left(\operatorname{Tor}_{i_{0}}^{R}(R / I, A)\right)$, we can check that

$$
\begin{aligned}
\widehat{\mathfrak{p}} & \in \operatorname{Var}\left(\operatorname{Ann}_{\widehat{R}} \operatorname{Tor}_{i_{0}}^{R}(R / I, A)\right) \\
& =\operatorname{Var}\left(\operatorname{Ann}_{\widehat{R}}\left(\operatorname{Ext}_{\widehat{R}}^{i_{0}}(\widehat{R} / I \widehat{R}, D(A))\right)\right) \\
& =\operatorname{Supp}_{\widehat{R}}\left(\operatorname{Ext}_{\widehat{R}}^{i_{0}}(\widehat{R} / I \widehat{R}, D(A)) .\right.
\end{aligned}
$$

Hence, $\operatorname{Ext}_{\widehat{R}_{\widehat{\mathfrak{p}}}}^{i_{0}}\left(\widehat{R}_{\widehat{\mathfrak{p}}} / I \widehat{R}_{\widehat{\mathfrak{p}}}, D(A)_{\widehat{\mathfrak{p}}}\right) \neq 0$. It follows that $\operatorname{depth}\left(I \widehat{R}_{\widehat{\mathfrak{p}}}, D(A)_{\widehat{\mathfrak{p}}}\right) \leq i_{0}$.

So $i_{0}=\operatorname{depth}\left(I \widehat{R}_{\widehat{\mathfrak{p}}}, D(A)_{\widehat{\mathfrak{p}}}\right)$.

Since $\widehat{\mathfrak{p}} \in \operatorname{Att}_{\widehat{R}}\left(\operatorname{Tor}_{i_{0}}^{R}(R / I, A)\right), \widehat{\mathfrak{p}} \in \operatorname{Ass}_{\widehat{R}}\left(\operatorname{Ext}_{\widehat{R}}^{i_{0}}(\widehat{R} / I \widehat{R}, D(A))\right)$. From this and by Lemma 3.1 we have

$$
\begin{aligned}
\widehat{\mathfrak{p}} \widehat{R}_{\widehat{\mathfrak{p}}} & \in \operatorname{Ass}_{\widehat{R}_{\widehat{\mathfrak{p}}}}\left(\operatorname{Ext}_{\widehat{R}_{\widehat{\mathfrak{p}}}}^{i_{0}}\left(\widehat{R}_{\widehat{\mathfrak{p}}} / I \widehat{R}_{\widehat{\mathfrak{p}}}, D(A)_{\widehat{\mathfrak{p}}}\right)\right) \\
& =\operatorname{Ass}_{\widehat{R}_{\widehat{\mathfrak{p}}}}\left(D(A)_{\widehat{\mathfrak{p}}} /\left(\bar{x}_{1}, \ldots, \bar{x}_{i_{0}}\right) D(A)_{\widehat{\mathfrak{p}}}\right) \cap \operatorname{Var}\left(I \widehat{R}_{\widehat{\mathfrak{p}}}\right) .
\end{aligned}
$$

Hence, $\widehat{\mathfrak{p}} \in \operatorname{Ass}_{\widehat{R}}\left(D(A) /\left(x_{1}, \ldots, x_{i_{0}}\right) D(A)\right) \cap \operatorname{Var}(I \widehat{R})$. It follows by Lemma 2.4 applying for the natural homomorphism $R \rightarrow \widehat{R}$, we get that

$$
\mathfrak{p} \in \operatorname{Ass}_{R}\left(D(A) /\left(x_{1}, \ldots, x_{i_{0}}\right) D(A)\right) \cap \operatorname{Var}(I) .
$$

Therefore we get the first inclusion

$$
\left(\bigcup_{i=0}^{t} \operatorname{Att}_{R}\left(\operatorname{Tor}_{i}^{R}(R / I, A)\right)\right)_{\geq k} \subseteq\left(\bigcup_{i=0}^{t} \operatorname{Ass}_{R}\left(D(A) /\left(x_{1}, \ldots, x_{i}\right) D(A)\right)\right)_{\geq k} \cap \operatorname{Var}(I) .
$$

Conversely, for any $\mathfrak{p} \in\left(\bigcup_{i=0}^{t} \operatorname{Ass}_{R} D(A) /\left(x_{1}, \ldots, x_{i}\right) D(A)\right)_{\geq k} \cap \operatorname{Var}(I)$, there exists an integer $i_{0} \leq t$ such that

$$
\mathfrak{p} \in \operatorname{Ass}_{R}\left(D(A) /\left(x_{1}, \ldots, x_{i_{0}}\right) D(A)\right)_{\geq k} \cap \operatorname{Var}(I)
$$

and

$$
\mathfrak{p} \notin \operatorname{Ass}_{R}\left(D(A) /\left(x_{1}, \ldots, x_{i}\right) D(A)\right)
$$

for all $i<i_{0}$. Hence, $\mathfrak{p} R_{\mathfrak{p}} \notin \operatorname{Ass}_{R_{\mathfrak{p}}}\left(D(A)_{\mathfrak{p}} /\left(x_{1}, \ldots, x_{i}\right) D(A)_{\mathfrak{p}}\right)$ for all $i<i_{0}$. Therefore

$$
\operatorname{Ass}_{R_{\mathfrak{p}}}\left(D(A)_{\mathfrak{p}} /\left(x_{1}, \ldots, x_{i}\right) D(A)_{\mathfrak{p}}\right)=\left(\operatorname{Ass}_{R_{\mathfrak{p}}}\left(D(A)_{\mathfrak{p}} /\left(x_{1}, \ldots, x_{i}\right) D(A)_{\mathfrak{p}}\right)\right)_{\geq 1}
$$


for all $i<i_{0}$. It follows that

$$
\operatorname{Ass}_{\widehat{R}_{\widehat{\mathfrak{p}}}}\left(D(A)_{\widehat{\mathfrak{p}}} /\left(\bar{x}_{1}, \ldots, \bar{x}_{i}\right) D(A)_{\widehat{\mathfrak{p}}}\right)=\left(\operatorname{Ass}_{\widehat{R}_{\widehat{\mathfrak{p}}}}\left(D(A)_{\widehat{\mathfrak{p}}} /\left(\bar{x}_{1}, \ldots, \bar{x}_{i}\right) D(A)_{\widehat{\mathfrak{p}}}\right)_{\geq 1}\right.
$$

Since $\bar{x}_{1}, \ldots, \bar{x}_{i_{0}}$ is an $D(A)_{\widehat{\mathfrak{p}}}$-poor filter sequence by Lemma 2.5 with notice that $x_{1}, \ldots, x_{i_{0}} \in I \widehat{R} \subseteq \widehat{\mathfrak{p}}$, it follows that $\bar{x}_{1}, \ldots, \bar{x}_{i_{0}}$ is an $D(A)_{\widehat{\mathfrak{p}}}$ regular sequence. Because $\mathfrak{p} \in \operatorname{Ass}_{R}\left(D(A) /\left(x_{1}, \ldots, x_{i_{0}}\right) D(A)\right) \cap \operatorname{Var}(I)$, we get

$$
\widehat{\mathfrak{p}} \widehat{R}_{\widehat{\mathfrak{p}}} \in \operatorname{Ass}_{\widehat{R}_{\widehat{\mathfrak{p}}}}\left(D(A)_{\widehat{\mathfrak{p}}} /\left(\bar{x}_{1}, \ldots, \bar{x}_{i_{0}}\right) D(A)_{\widehat{\mathfrak{p}}}\right) \cap \operatorname{Var}\left(I \widehat{R}_{\widehat{\mathfrak{p}}}\right) .
$$

From this we obtain

$$
0=\operatorname{depth}\left(I \widehat{R}_{\widehat{\mathfrak{p}}}, D(A)_{\widehat{\mathfrak{p}}} /\left(\bar{x}_{1}, \ldots, \bar{x}_{i_{0}}\right) D(A)_{\widehat{\mathfrak{p}}}\right)=\operatorname{depth}\left(I \widehat{R}_{\widehat{\mathfrak{p}}}, D(A)_{\widehat{\mathfrak{p}}}\right)-i_{0}
$$

It implies that $\operatorname{depth}\left(I \widehat{R}_{\widehat{\mathfrak{p}}}, D(A)_{\widehat{\mathfrak{p}}}\right)=i_{0}$. By Lemma 3.1 , we have the equality

$$
\begin{aligned}
& \operatorname{Ass}_{\widehat{R}_{\widehat{\mathfrak{p}}}}\left(D(A)_{\widehat{\mathfrak{p}}} /\left(\bar{x}_{1}, \ldots, \bar{x}_{i_{0}}\right) D(A)_{\widehat{\mathfrak{p}}}\right) \cap \operatorname{Var}\left(I \widehat{R}_{\widehat{\mathfrak{p}}}\right) \\
= & \operatorname{Ass}_{\widehat{R}_{\widehat{\mathfrak{p}}}}\left(\operatorname{Ext}_{\widehat{R}_{\widehat{\mathfrak{p}}}}^{i_{0}}\left(\widehat{R}_{\widehat{\mathfrak{p}}} / I \widehat{R}_{\widehat{\mathfrak{p}}}, D(A)_{\widehat{\mathfrak{p}}}\right)\right) .
\end{aligned}
$$

Therefore $\widehat{\mathfrak{p}} \widehat{R}_{\widehat{\mathfrak{p}}} \in \operatorname{Ass}_{\widehat{R}_{\widehat{\mathfrak{p}}}}\left(\operatorname{Ext}_{\widehat{R}_{\widehat{\mathfrak{p}}}}^{i_{0}}\left(\widehat{R}_{\widehat{\mathfrak{p}}} / I \widehat{R}_{\widehat{\mathfrak{p}}}, D(A)_{\widehat{\mathfrak{p}}}\right)\right)$. Hence

$$
\widehat{\mathfrak{p}} \in \operatorname{Ass}_{\widehat{R}}\left(\operatorname{Ext}_{\widehat{R}}^{i_{0}}(\widehat{R} / I \widehat{R}, D(A))\right) .
$$

From this we have $\widehat{\mathfrak{p}} \in \operatorname{Att}_{\widehat{R}}\left(\operatorname{Tor}_{i_{0}}^{R}(R / I, A)\right)$. Hence $\mathfrak{p} \in \operatorname{Att}_{R}\left(\operatorname{Tor}_{i_{0}}^{R}(R / I, A)\right)$ and we get the inclusion

$\left(\bigcup_{i=0}^{t} \operatorname{Ass}_{R}\left(D(A) /\left(x_{1}, \ldots, x_{i}\right) D(A)\right)\right)_{\geq k} \cap \operatorname{Var}(I) \subseteq\left(\bigcup_{i=0}^{t} \operatorname{Att}_{R}\left(\operatorname{Tor}_{i}^{R}(R / I, A)\right)\right)_{\geq k}$. Hence,

$\left(\bigcup_{i=0}^{t} \operatorname{Ass}_{R}\left(D(A) /\left(x_{1}, \ldots, x_{i}\right) D(A)\right)\right)_{\geq k} \cap \operatorname{Var}(I)=\left(\bigcup_{i=0}^{t} \operatorname{Att}_{R}\left(\operatorname{Tor}_{i}^{R}(R / I, A)\right)\right)_{\geq k}$.

The claim is proved.

Next, we prove the first equality of Theorem 3.4. Let

$$
\mathfrak{p} \in\left(\bigcup_{i=0}^{t} \operatorname{Att}_{R}\left(\operatorname{Tor}_{i}^{R}(R / I, A)\right)\right)_{\geq k}
$$

We take an integer $i_{0} \leq t$ such that

$$
\mathfrak{p} \in \operatorname{Att}_{R}\left(\operatorname{Tor}_{i_{0}}^{R}(R / I, A)\right) \text { and } \mathfrak{p} \notin \operatorname{Att}_{R}\left(\operatorname{Tor}_{i}^{R}(R / I, A)\right)
$$

for all $i<i_{0}$.

It follows by the claim that $\mathfrak{p} \notin \operatorname{Ass}_{R}\left(D(A) /\left(x_{1}, \ldots, x_{i}\right) D(A)\right)$ for all $i<i_{0}$, where $\left(x_{1}, \ldots, x_{r}\right)$ is an $A$-cosequence in dimension $>k$ in $I$. From this and by similar arguments as in the proof of the claim, we get the fact that $i_{0}=$ $\operatorname{depth}\left(I \widehat{R}_{\widehat{\mathfrak{p}}}, D(A)_{\widehat{\mathfrak{p}}}\right)$. 
Since $\widehat{\mathfrak{p}} \in \operatorname{Att}_{\widehat{R}}\left(\operatorname{Tor}_{i_{0}}^{R}(R / I, A)\right)$, we get $\widehat{\mathfrak{p}} \in \operatorname{Ass}_{\widehat{R}}\left(\operatorname{Ext}_{\widehat{R}}^{i_{0}}(\widehat{R} / I \widehat{R}, D(A))\right)$. Hence,

$$
\widehat{\mathfrak{p}} \widehat{R}_{\widehat{\mathfrak{p}}} \in \operatorname{Ass}_{\widehat{R}_{\widehat{\mathfrak{p}}}}\left(\operatorname{Ext}_{\widehat{R}_{\widehat{\mathfrak{p}}}}^{i_{0}}\left(\widehat{R}_{\widehat{\mathfrak{p}}} / I \widehat{R}_{\widehat{\mathfrak{p}}}, D(A)_{\widehat{\mathfrak{p}}}\right)\right)
$$

It follows by Remark 3.2 that

$$
\begin{aligned}
\widehat{\mathfrak{p}} \widehat{R}_{\widehat{\mathfrak{p}}} & \in \operatorname{Ass}_{\widehat{R}_{\widehat{\mathfrak{p}}}}\left(H_{I \widehat{R}_{\widehat{\mathfrak{p}}}}^{i_{0}}\left(D(A)_{\widehat{\mathfrak{p}}}\right)\right) \\
& =\operatorname{Ass}_{\widehat{R}_{\widehat{\mathfrak{p}}}}\left(H_{I^{n} \widehat{R}_{\widehat{\mathfrak{p}}}}^{i_{0}}\left(D(A)_{\widehat{\mathfrak{p}}}\right)\right) \\
& =\operatorname{Ass}_{\widehat{R}_{\widehat{\mathfrak{p}}}}\left(\operatorname{Ext}_{\widehat{R}_{\widehat{\mathfrak{p}}}}^{i_{0}}\left(\widehat{R}_{\widehat{\mathfrak{p}}} / I^{n} \widehat{R}_{\widehat{\mathfrak{p}}}, D(A)_{\widehat{\mathfrak{p}}}\right)\right) .
\end{aligned}
$$

Hence, $\widehat{\mathfrak{p}} \in \operatorname{Ass}_{\widehat{R}}\left(\operatorname{Ext}_{\widehat{R}}^{i_{0}}\left(\widehat{R} / I^{n} \widehat{R}, D(A)\right)\right)$. So, $\widehat{\mathfrak{p}} \in \operatorname{Att}_{\widehat{R}}\left(\operatorname{Tor}_{i_{0}}^{R}\left(R / I^{n}, A\right)\right)$. By Lemma 2.3, $\mathfrak{p} \in \operatorname{Att}_{R} \operatorname{Tor}_{i_{0}}^{R}\left(R / I^{n}, A\right)$. From this we obtain the inclusion

$$
\left(\bigcup_{i=0}^{t} \operatorname{Att}_{R}\left(\operatorname{Tor}_{i}^{R}(R / I, A)\right)\right)_{\geq k} \subseteq\left(\bigcup_{i=0}^{t} \operatorname{Att}_{R}\left(\operatorname{Tor}_{i}^{R}\left(R / I^{n}, A\right)\right)\right)_{\geq k} .
$$

Now by Lemma 3.3 we obtain the first equality

$$
\left(\bigcup_{i=0}^{t} \operatorname{Att}_{R}\left(\operatorname{Tor}_{i}^{R}(R / I, A)\right)\right)_{\geq k}=\left(\bigcup_{i=0}^{t} \operatorname{Att}_{R}\left(\operatorname{Tor}_{i}^{R}\left(R / I^{n}, A\right)\right)\right)_{\geq k} .
$$

Similarly, we have the second equality

$$
\left(\bigcup_{i=0}^{t} \operatorname{Att}_{R}\left(\operatorname{Tor}_{i}^{R}(R / I, A)\right)\right)_{\geq k}=\left(\bigcup_{i=0}^{t} \operatorname{Att}_{R}\left(\operatorname{Tor}_{i}^{R}\left(R /\left(a_{1}^{n_{1}}, \ldots, a_{l}^{n_{l}}\right), A\right)\right)\right)_{\geq k} .
$$

The proof is completed.

Remark 3.5. Theorem 3.4 may be stated as follows: Let $k \geq 0$ be an integer and $r=\operatorname{Width}_{>k}(I, A)$. Then, for all $t \leq r$, the followings are true.

(i) $\left(\bigcup_{i=0}^{t} \operatorname{Att}_{R}\left(\operatorname{Tor}_{i}^{R}\left(R / I^{n}, A\right)\right)\right)_{\geq k}$ is independent of the choice of $n$.

(ii) $\left(\bigcup_{i=0}^{t} \operatorname{Att}_{R}\left(\operatorname{Tor}_{i}^{R}\left(R /\left(a_{1}^{n_{1}}, \ldots, a_{l}^{n_{l}}\right), A\right)\right)\right)_{\geq k}$ is independent of the choice of $n_{1}, \ldots, n_{l}$ for any system of generators $\left(a_{1}, \ldots, a_{l}\right)$ of $I$.

In the case $k=0$, by Remark 3.5 we get the following result.

Corollary 3.6. Let $r=\mathrm{Width}_{>0}(I, A)$. Then, for all $t \leq r$, the followings are true.

(i) $\bigcup_{i=0}^{t} \operatorname{Att}_{R}\left(\operatorname{Tor}_{i}^{R}\left(R / I^{n}, A\right)\right)$ is independent of the choice of $n$.

(ii) $\bigcup_{i=0}^{t} \operatorname{Att}_{R}\left(\operatorname{Tor}_{i}^{R}\left(R /\left(a_{1}^{n_{1}}, \ldots, a_{l}^{n_{l}}\right), A\right)\right)$ is independent of the choice of $n_{1}, \ldots, n_{l}$ for any system of generators $\left(a_{1}, \ldots, a_{l}\right)$ of $I$. 
Now we consider the case $k=-1$. Firstly we recall a result of Nhan and Dung in [7].

Lemma $3.7\left(\left[7\right.\right.$, Lemma 3.5]). Set $P_{t}=\bigcup_{i=0}^{t-1} \operatorname{Var}\left(\operatorname{Ann}_{R}\left(\operatorname{Tor}_{i}^{R}(R / I, A)\right)\right)$, where $t$ is a positive integer. Then

$$
\begin{aligned}
& \operatorname{Att}_{R}\left(\operatorname{Tor}_{t}^{R}\left(R / I^{n}, A\right)\right) \cup P_{t} \\
= & \operatorname{Att}_{R}\left(\operatorname{Tor}_{t}^{R}\left(R /\left(a_{1}^{n_{1}}, \ldots, a_{l}^{n_{l}}\right), A\right)\right) \cup P_{t} \\
= & \operatorname{Att}_{R}\left(\operatorname{Tor}_{t}^{R}(R / I, A)\right) \cup P_{t}
\end{aligned}
$$

for any system of generators $\left(a_{1}, \ldots, a_{l}\right)$ of $I$ and all positive integer $n, n_{1}$, $\ldots, n_{l}$.

Proposition 3.8. Let $r=\operatorname{Width}(I, A)$. Then the followings are true.

(i) $\operatorname{Att}_{R}\left(\operatorname{Tor}_{r}^{R}\left(R / I^{n}, A\right)\right)$ is independent of the choice of $n$.

(ii) $\operatorname{Att}_{R}\left(\operatorname{Tor}_{r}^{R}\left(R /\left(a_{1}^{n_{1}}, \ldots, a_{l}^{n_{l}}\right), A\right)\right)$ is independent of the choice of $n_{1}$, $\ldots, n_{l}$ for any system of generators $\left(a_{1}, \ldots, a_{l}\right)$ of $I$.

Proof. By [7, Theorem 2.8], we have $\operatorname{Tor}_{i}^{R}(R / I, A)=0$ for all $i<r$. Hence $P_{r}=\bigcup_{i=0}^{r-1} \operatorname{Var}\left(\operatorname{Ann}_{R}\left(\operatorname{Tor}_{i}^{R}(R / I, A)\right)\right)=\emptyset$. It follows by Lemma 3.7 that

$$
\begin{aligned}
\operatorname{Att}_{R}\left(\operatorname{Tor}_{r}^{R}(R / I, A)\right) & =\operatorname{Att}_{R}\left(\operatorname{Tor}_{r}^{R}\left(R / I^{n}, A\right)\right) \\
& =\operatorname{Att}_{R}\left(\operatorname{Tor}_{r}^{R}\left(R /\left(a_{1}^{n_{1}}, \ldots, a_{l}^{n_{l}}\right), A\right)\right) .
\end{aligned}
$$

The proof is completed.

\section{References}

[1] M. Brodmann, Asymptotic stability of $\operatorname{Ass}_{R}\left(M / I^{n} M\right)$, Proc. Amer. Math. Soc. 74 (1979), no. 1, 16-18.

[2] M. Brodmann and R. Y. Sharp, Local Cohomology: an algebraic introduction with geometric applications, Cambridge University Press, 1998.

[3] N. T. Cuong, P. Schenzel, and N. V. Trung, Verallgemeinerte Cohen-Macaulay Moduln, Math. Nachr. 85 (1978), 57-73.

[4] M. Katzman, An example of an infinite set of associated primes of a local cohomology module, J. Algebra 252 (2002), no. 1, 161-166.

[5] I. G. Macdonald, Secondary representation of modules over a commutative ring, Symposia Mathematica, Vol. XI (Convegno di Algebra Commutativa, INDAM, Rome, 1971), pp. 23-43. Academic Press, London, 1973.

[6] L. Melkersson and P. Schenzel, Asymptotic prime ideals related to derived functions, Proc. Amer. Math. Soc. 117 (1993), no. 4, 935-938.

[7] L. T. Nhan and N. T. Dung, A Finiteness Result for Attached Primes of Certain TorModules, Algebra Colloq. 19 (2012), 787-796.

[8] L. T. Nhan and N. V. Hoang, A finiteness result for attached primes of Artinian local cohomology, J. Algebra Appl. 13 (2014), no. 1, 1350063, 14 pp.

[9] A. Ooishi, Matlis duality and the width of a module, Hiroshima Math. J. 6 (1976), no. $3,573-587$. 
[10] L. J. Ratliff, On prime divisors of $I^{n}, n$ large, Michigan Math. J. 23 (1976), no. 4, 337-352.

[11] R. Y. Sharp, Some results on the vanishing of local cohomology modules, Proc. Lond. Math. Soc. 30 (1975), 177-195.

[12] _ Asymptotic behaviour of certain sets of attached prime ideals, J. Lond. Math. Soc. 34 (1986), no. 2, 212-218.

DEPARTMENT OF MATHEMATICS

TAY NGUYEN University

DAK LAK, VIET NAM

E-mail address: phkhanhlt@gmail.com 